\title{
LA "VENTAJA DEL SÍNDROME DE DOWN" EN LA CALIDAD DE VIDA DE JÓVENES CON DISCAPACIDAD INTELECTUAL
}

\section{The "Down Syndrome Advantage" in the Young People with Intellectual Disabilities Quality of Life}

\author{
Ana Berástegui \\ Universidad Pontificia Comillas \\ a.berastegui@comillas.edu \\ Sara Corral \\ Universidad Pontificia Comillas
}

Recepción: 22 de enero de 2020

Aceptación definitiva: 6 de julio de 2020

Resumen: El objetivo del estudio fue examinar el fenómeno descrito en la literatura como la "ventaja del síndrome de Down" en la calidad de vida familiar e individual de jóvenes con discapacidad intelectual (DI). La muestra la formaron 84 jóvenes con DI (Edad media 21,41 años; DT =2,87), de los que 20 tenían síndrome de Down (SD) $(23,81 \%)$. Los instrumentos utilizados fueron la Escala de Calidad de Vida Familiar (CVF) y la Escala INICO-FEAPS, en su versión informada por otros (CVI-H) y autoinforme (CVI-A). Los resultados reflejan mayores puntuaciones en CVF y CVI-H en las familias de los jóvenes con SD y menores en CVI-A que en los jóvenes sin SD. Ni la edad de los padres ni el nivel de ingresos se relacionaron con estas variables. Que las diferencias en calidad de vida familiar e individual se den cuando informan los progenitores, pero se inviertan en la versión autoinformada, sugiere una explicación del fenómeno no causal sino relacionada con las expectativas familiares y su acceso a recursos y apoyos, y nos impulsa a dar importancia a la visión de la persona en la evaluación de la calidad de vida de los jóvenes con DI.

Palabras clave: calidad de vida familiar; calidad de vida individual; ventaja del síndrome de Down; discapacidad intelectual.

Aвstract: The aim of the study was to examine the "Down syndrome advantage" phenomenon in the family and individual quality of life of young people with intellectual disabilities (ID). The sample consisted of 84 young people with ID (mean age 21,41 years; $\mathrm{SD}=2,87), 20$ of them with Down syndrome (DS) $(23,81 \%)$. The instruments were the 


\section{LA “VENTAJA DEL SÍNDROME DE DOWN” EN LA CALIDAD DE VIDA DE JÓVENES CON DISCAPACIDAD INTELECTUAL ANA BERÁSTEGUI Y SARA CORRAL}

Family Quality of Life Scale (FQoL) and the INICO-FEAPS Scale, in its reported by others version $(\mathrm{QoL}-\mathrm{H})$ and self-reported version $(\mathrm{QoL}-\mathrm{A})$. The results reflect greater satisfaction with FQoL and better QoL-H scores in the young people with DS' families, while QoL-A is higher among young people without DS. Neither the age of the parents nor the level of income was related to these variables. The fact that the differences found in family and individual QoL are favorable for young people with DS when their relatives are the informants, but unfavorable in the self-reported version, suggests an not causal explanation of the phenomenon, but related to family expectations and their access to resources and supports. This encourages us to highlight the importance of people's self-perception and report in the evaluation of the QoL of young people with ID.

KEY WORDs: family quality of life; individual quality of life; Down syndrome advantage; intellectual disability.

\section{Introducción}

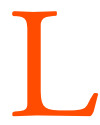

A “ventaja del síndRome De Down” es un fenómeno descrito en la literatura, que encuentra consistentemente mejores resultados en diferentes variables de funcionamiento y bienestar familiar en las familias que tienen un miembro con $\mathrm{SD}$, en comparación con familias que tienen un miembro con otro tipo de discapacidad intelectual -DI- (Glidden, Grein y Ludwig, 2014; Hodap, 2007; Hodapp, Burke y Urbano, 2012; Mitchell, Hauser-Cram y Crossman, 2015). La investigación sobre este fenómeno busca comprender si estas diferencias se mantienen en distintas variables y distintas muestras, cuál es su origen o si son un artefacto del impacto de otras variables sociodemográficas.

Las variables en las que se han encontrado mejores resultados para las familias con SD han estado muy relacionadas con la calidad de vida familiar (CVF), por ejemplo, bienestar familiar (Abbeduto et al., 2004; Dabrowska y Pisula, 2010), satisfacción familiar (Brown, MacAdam-Crisp, Wang e Iarocci, 2006), estrés familiar (Phillips, Conners y Curtner-Smith, 2017), optimismo (Poehlmann, Clements, Abbeduto y Farsad, 2005) o interacción familiar positiva (Mitchell et al., 2015).

Además, se han encontrado en variables individuales de distintos miembros de la familia como madres (Fidler, Hodapp y Dykens, 2000; Griffith, Hastings, Nash y Hill, 2010; Hodapp, Ricci, Ly y Fidler, 2003; Tekinarslan, 2013), padres (Abbeduto et al., 2004; Dabrowska y Pisula, 2010; Hartley, Seltzer, Head y Abbeduto, 2012; Ricci y Hodapp, 2003; Stoneman, 2007) y hermanos (Fisman, Wolf, Ellison y Freeman, 2000; Shivers, McGregor y Hough, 2019).

Aunque la mayoría de estos estudios se han hecho en familias con niños pequeños (Bertoli et al., 2011), se obtienen resultados semejantes cuando los hijos son adolescentes o adultos (Harektley, Seltzer, Head y Abbeduto, 2012), aunque parece un fenómeno no del todo estable en el tiempo (Glidden et al., 2014; Hodapp, 2007).

Estas diferencias a favor de las familias con SD se han encontrado comparando con familiares de personas con otros diagnósticos como parálisis cerebral (Lee, Burke, Arnold y Owen, 2018; Tekinarslan, 2013), síndrome de Willams (Fidler 
et al., 2000), síndrome de X-Frágil (Abbeduto et al., 2004), síndrome de Prader Willy (Rosner, Hodapp, Fidler, Sagun y Dykens, 2004) o trastorno del espectro autista (TEA) (Abbeduto et al., 2004; Blacher y McIntyre, 2006; Brown et al., 2006; Pisula, 2007). Algunos estudios encuentran que la diferencia solo es evidente cuando se compara a familias con hijos con SD con familias con hijos con TEA, lo que algún autor ha etiquetado como la "desventaja del autismo" (Hodapp et al., 2003; Stoneman, 2007).

Sin embargo, cuando se compara a las familias de hijos con SD con aquellas cuyos hijos no tienen discapacidad, se encuentran peores resultados en variables como el estrés familiar (Phillips et al., 2017) o calidad de vida familiar (Brown et al., 2006; Wiley y Renk, 2007), lo que algunos autores han descrito como "desventaja del SD" (Acharya, 2011).

Al intentar explicar el origen de "la ventaja del SD" se ha acudido a la explicación fenotípica, aludiendo a que las personas con SD tienen menores problemas de comportamiento y problemas de adaptación que las personas con otras discapacidades intelectuales (Baker y Kaladjian, 2013; Blacher, Foley et al., 2016; Ricci y Hodapp, 2003) y más competencias sociales (Kasari y Freeman, 2001; Rosner et al., 2004), lo que haría que la experiencia familiar fuera menos estresante.

Otra explicación se basa en que el SD es probablemente el síndrome mejor conocido socialmente, lo que promueve conductas positivas por parte de la población, una mayor presencia de apoyos naturales y un acceso más fácil a diversas organizaciones que proporcionan apoyo e información en comparación con otras discapacidades. Este mayor conocimiento social no solo incrementa el apoyo social formal e informal para estas familias (Hodap, 2007; Lee et al., 2018; Poehlmann et al., 2005); sino que mejora las propias expectativas familiares con respecto a sus hijos (Rodgers, 2008).

Por último, se ha valorado que esta diferencia puede ser el artefacto de otras variables como la mayor edad de las madres o los mejores niveles de renta en familias con hijos con SD, de tal manera que estas diferencias desaparecerían al controlar dichas variables (Abbeduto et al., 2004; Blacher y McIntyre, 2006; Corrice y Glidden, 2009; Esbensen y Seltzer, 2011; Glidden et al., 2014; Stoneman, 2007).

La investigación sobre la calidad de vida en las familias con hijos con discapacidad intelectual, y el posible impacto diferencial de los distintos diagnósticos, es fundamental, pero la dispersión en las variables de estudio puede estar ensombreciendo los resultados, por lo que el uso del concepto de CVF, que engloba muchas de las cuestiones recogidas, resulta especialmente útil. Park et al. (2003) definen la calidad de vida familiar como el grado en el que los miembros de una familia tienen sus necesidades cubiertas, pueden disfrutar de su vida juntos y cuentan con oportunidades para perseguir y alcanzar metas que son trascendentales para ellos.

Por otra parte, a pesar de la extensión de la investigación precedente sobre las variables familiares, existe muy poca literatura sobre estas diferencias en la calidad de vida de las personas con DI, con y sin SD. La investigación sobre calidad de vida individual se ha convertido en una de las variables clave para la intervención en DI. Sin embargo, no se ha estudiado en profundidad el fenómeno de la "ventaja del SD" en esta variable. El concepto de calidad de vida individual se define como un estado 


\section{LA “VENTAJA DEL SÍNDROME DE DOWN” EN LA CALIDAD DE VIDA DE JÓVENES CON DISCAPACIDAD INTELECTUAL \\ ANA BERÁSTEGUI Y SARA CORRAL}

de bienestar personal que: a) es multidimensional; b) tiene propiedades universales y ligadas a la cultura; c) tiene componentes objetivos y subjetivos; y d) está influenciado por características personales y factores ambientales (Schalock, Keith, Verdugo y Gómez, 2010; Schalock y Verdugo, 2012).

La medida de la CVI se ha apoyado mayoritariamente en el informe de otros, siendo los padres y profesores los principales informantes. Esto puede deberse a que se ha investigado esta cuestión más frecuentemente con niños y adolescentes que con adultos (Bertoli et al., 2011). Cuando se ha estudiado la CV en adultos, se ha encontrado que los adultos con SD tienen más capacidades funcionales, menos problemas de comportamiento, menos analfabetismo, más amigos, más independencia residencial y unas necesidades de apoyo mejor cubiertas comparado con los adultos con TEA (Esbensen, Bishop, Sltzer, Greenberg y Taylor, 2010).

El propósito principal de este estudio es determinar si hay diferencias en la calidad de vida de las personas con SD frente a aquellas con otras DI en una muestra de jóvenes. Específicamente, si las diferencias aparecen en la CVF o en la CVI, y si estas últimas se mantienen tanto cuando es informada por la familia (CVI-O) como cuando es informada por la propia persona (CVI-A). Asimismo, se estudiará el impacto de variables sociodemográficas como la edad de la madre o el nivel de ingresos familiares en estas diferencias.

\section{Método}

\subsection{Participantes}

La muestra del estudio la formaron un total de 84 jóvenes con discapacidad. Las personas con DI pertenecían al programa DEMOS de la Universidad Pontificia de Comillas, un proyecto de formación para el empleo e inclusión universitaria para jóvenes con discapacidad intelectual (Berástegui, García y López-Rioboo, 2015). De estas, un 51,20 \% de la muestra eran mujeres y un 48,8 \% hombres, con edades comprendidas entre los 18 y los 35 años, situándose la media de edad en los 21,41 años $(D T=2,87)$. De ellos, 20 tenían SD y 64 tenían discapacidad intelectual de otras etiologías (trastorno del espectro del autismo, parálisis cerebral, síndrome de Sotos y otros trastornos del desarrollo neurológico no especificado). Del grupo con SD, la media de edad era de 21,55 años $(D T=0,93)$, siendo un $40 \%$ hombres y un $60 \%$ mujeres. Respecto al grupo sin SD, la media de edad era de 21,38 años $(D T=3,17)$, siendo más hombres $-51,50 \%$ - que mujeres $-48,50 \%-$.

Respecto a los progenitores que participaron en el estudio, un $26 \%$ de los participantes fueron padres y un $74 \%$ madres, con edades comprendidas entre 39 y 72 años, siendo la media de edad de 56,53 años $(D T=6,31)$. En cuanto a los ingresos familiares, el 11,1 \% tuvo unos ingresos familiares anuales inferiores a 15.000 euros, un $19,4 \%$ entre $15.000 €$ y $24.000 €$, un $37,5 \%$ entre $25.000 €$ y $49,000 €$ y un $31,9 \%$ superiores a 49.000 euros. 


\subsection{Instrumentos}

Escala de Calidad de Vida Familiar (ECVF) (Verdugo, Rodríguez y Sainz, 2009). Es la adaptación española de la "Family Quality of Life Survey", desarrollada en el Beach Center. Está formada por tres secciones: una primera que recoge los datos sociodemográficos; una segunda en la que se evalúan la importancia y la satisfacción con los apoyos y servicios recibidos, y una tercera en la que se evalúa la calidad de vida familiar propiamente dicha (CVF) y que es la utilizada en esta investigación. Esta sección consta de 25 ítems que evalúan los niveles de importancia y satisfacción en 5 dimensiones de CVF: interacción familiar (IF), papel de los padres (PP), bienestar emocional (BE), bienestar físico y material (BFM) y recursos y apoyos relacionados con la persona con discapacidad (RyA). En esta investigación usamos la escala de satisfacción, que ofrece cinco opciones de respuesta (de 1 "muy insatisfecho" a 5 "muy satisfecho"). La escala fue validada en población colombiana con buenos índices de fiabilidad test rest $(\mathrm{r}=$ $0,78)$ y una consistencia interna de 0,95 para la escala total $(\mathrm{BF}=0,85 ; \mathrm{RyA}=0,87 ; \mathrm{IF}=$ 0,87; PP = 0,85; BFM = 0,87; Verdugo, Córdoba y Gómez, 2006). La escala tiene un alfa de Cronbach en esta muestra de 0,93.

Escala INICO-FEAPS de Evaluación Integral de la Calidad de Vida de Personas con Discapacidad Intelectual o del Desarrollo (Verdugo, Gómez, Arias, Santamaría, Clavero y Tamarit, 2013). Se trata de una escala tipo Likert compuesta de 72 ítems con cuatro opciones de respuesta ("nunca", "algunas veces", "frecuentemente” y "siempre”). Los ítems se agrupan en 8 subescalas congruentes con el modelo de calidad de vida de Schalock y Verdugo (2012): autodeterminación, derechos, bienestar emocional, inclusión social, desarrollo personal, relaciones interpersonales, bienestar material y bienestar físico. Consta de dos versiones: un informe de otras personas o heteroinforme -completado por otra persona cercana a esta: profesional, familiar, tutor, etc.- (CVI-H) y un autoinforme -completado por la persona con discapacidad intelectual- (CVI-A). La subescala autoinforme se ha diseñado para aplicarla a personas con DI con adecuadas habilidades de lenguaje receptivo y expresivo. La escala original cuenta con un alfa de Cronbach de 0,94 en la versión "Informe de otras personas"; y un 0,89 en la versión "Autoinforme" y adecuadas consistencias para todas las subescalas (Tabla 1). En nuestra aplicación la escala CVI-H alcanza una consistencia interna de 0,91 y la de CVI-A de 0,85.

TABLA 1. Índices de fiabilidad alpha de Cronbach para las distintas escalas de la escala INICO-FEAPS (Verdugo et al., 2013)

\begin{tabular}{|l|c|c|}
\hline & Autoinforme & Informe de otros \\
\hline Autodeterminación & 0,652 & 0,804 \\
\hline Derechos & 0,86 & 0,694 \\
\hline Bienestar emocional & 0,666 & 0,792 \\
\hline Inclusión social & 0,567 & 0,723 \\
\hline Desarrollo personal & 0,646 & 0,703 \\
\hline Relaciones interpersonales & 0,65 & 0,798 \\
\hline Bienestar material & 0,615 & 0,703 \\
\hline Bienestar físico & 0,525 & 0,659 \\
\hline
\end{tabular}




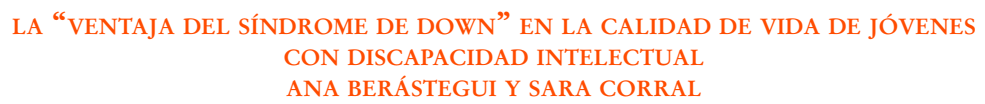

\subsection{Procedimiento}

El método de muestreo fue intencional o deliberado, no probabilístico, seleccionando de forma directa a los sujetos de la investigación. Respecto a la dimensión ética del estudio, la participación fue voluntaria, no estuvo retribuida y previamente a su participación se solicitó el consentimiento informado tanto a los participantes como a sus familiares. Todos los jóvenes adultos convivían con el progenitor que respondía a la encuesta. Asimismo, se informó de los procedimientos para proteger la información de tal manera que se asegurara el anonimato y la confidencialidad de todos los participantes.

La recogida de datos se llevó a cabo como parte del proceso de admisión al programa DEMOS. Uno de los progenitores, indistintamente, contestó las escalas de CVF y CVI-H en su domicilio. La aplicación de las escalas de CVI-A se realizó por personal cualificado perteneciente a dicho instituto, de forma individual y sin límite de tiempo, prestando el apoyo necesario para su cumplimentación, siguiendo las instrucciones del manual de aplicación (Verdugo et al., 2013).

\subsection{Análisis de datos}

Se realizaron análisis descriptivos y de frecuencias para describir las características sociodemográficas de los participantes.

Se comprobaron los supuestos de normalidad y, al no cumplirse, se realizaron pruebas no paramétricas para llevar a cabo los análisis estadísticos. Por tanto, para analizar las diferencias en calidad de vida entre jóvenes con y sin SD se utilizó la prueba no paramétrica de U de Mann-Whitney. Se calcularon los tamaños del efecto para los contrastes significativos según la fórmula $r=Z / \sqrt{ } N$ (Tomczak y Tomcak, 2014), que se interpreta como pequeño $(r=0,1-0,3)$, moderado $(r=0,3-0,5)$, grande $(r=0,5-0,7)$ o muy grande $(r=0,7-1)$ siguiendo la propuesta de Cohen (1988). Finalmente, se realizó un ANCOVA para determinar si las variables de ingresos y de edad parental estaban relacionadas con las variables de calidad de vida. Los contrastes se han realizado con un nivel de confianza del $95 \%$.

Para realizar los análisis estadísticos se utilizó el paquete estadístico SPSS -22-.

\section{Resultados}

\subsection{CVF en jóvenes con y $\sin S D$}

La calidad de vida familiar de los jóvenes con discapacidad intelectual, informada por sus progenitores, presenta diferencias significativas entre los que tienen SD y los que no $(U=-294,00 ; p=0,03 ; r=0,30)$, siendo mayor la satisfacción con la calidad de vida familiar de las familias con hijos con SD que la de aquellas con otras discapacidades y el tamaño del efecto mediano (Tabla 2). Estas diferencias solo se mantienen para 


\section{LA “VENTAJA DEL SÍNDROME DE DOWN” EN LA CALIDAD DE VIDA DE JÓVENES CON DISCAPACIDAD INTELECTUAL \\ ANA BERÁSTEGUI Y SARA CORRAL}

la subescala de satisfacción con los recursos o apoyos relacionados con la persona con discapacidad que también son mayores para las familias con miembros con $\mathrm{SD}$, aunque el tamaño del efecto es entre pequeño y moderado $(U=369,00 ; p=0,03 ; r=0,29)$.

\begin{tabular}{|c|c|c|c|c|c|c|}
\hline \multicolumn{7}{|c|}{$\begin{array}{c}\text { TABLA 2. U de Mann Whitney, media y desviación típica de la calidad de vida } \\
\text { individual autoinformada }\end{array}$} \\
\hline & \begin{tabular}{|c|}
$S D$ \\
\end{tabular} & No SD & \multirow{2}{*}{ Rango } & \multirow{2}{*}{$U$} & \multirow{2}{*}{$Z$} & \multirow{2}{*}{$p$} \\
\hline & $M \pm D T$ & $M \pm D T$ & & & & \\
\hline Calidad de vida familiar & $108,00 \pm 11,60$ & $96,07 \pm 17,05$ & $25-125$ & 294,00 & 2,11 & 0,03 \\
\hline Bienestar emocional & $15,40 \pm 2,37$ & $13,39 \pm 3,73$ & $4-20$ & 288,00 & 1,52 & 0,13 \\
\hline Recursos o apoyos & $18,27 \pm 2,05$ & $15,5 \pm 3,41$ & $4-20$ & 369,00 & 2,22 & 0,03 \\
\hline Interacción familiar & $26,00 \pm 4,27$ & $23,55 \pm 5,41$ & $6-30$ & 339,50 & 1,34 & 0,18 \\
\hline Papel de padres & $25,60 \pm 4,50$ & $23,33 \pm 4,51$ & $6-30$ & 317,00 & 1,46 & 0,14 \\
\hline Bienestar fco. y material & $21,55 \pm 2,38$ & $21,09 \pm 3,56$ & $5-25$ & 255,50 & $-0,06$ & 0,95 \\
\hline
\end{tabular}

*Nota: $\mathrm{M}=$ Media; DT = Desviación típica $\mathrm{SD}=$ Síndrome de Down.

\subsection{CVI-H en jóvenes con y sin SD}

En relación con la calidad de vida individual informada por un progenitor, se encuentran puntuaciones significativamente mayores en los jóvenes con DI y SD que en los que no tienen $\mathrm{SD}(U=2,36 ; p=0,018 ; r=0,31)$, siendo el tamaño del efecto moderado. Estas diferencias se mantienen en las subescalas desarrollo personal $(U=-2,57$; $p=0,01 ; r=0,34)$ y bienestar físico $(U=-2,76 ; p=0,01 ; r=0,36)$, con tamaños del efecto moderados, y de bienestar emocional $(U=-1,74 ; p=0,08 ; r=0,23)$, con un tamaño del efecto pequeño (ver Tabla 3 ).

TABla 3. U de Mann Whitney, media y desviación típica de la calidad de vida individual informada por otros

\begin{tabular}{|l|c|c|c|c|c|c|}
\hline & $S D$ & No $S D$ & \multirow{2}{*}{ Rango } & $U$ & $Z$ & $p$ \\
\cline { 2 - 6 } & $M \pm D T$ & $M \pm D T$ & & & \\
\hline Calidad de vida & $3,51 \pm 0,23$ & $3,27 \pm 0,27$ & $72-288$ & 122,5 & $-2,36$ & 0,01 \\
\hline Autodeterminación & $3,27 \pm 0,34$ & $2,95 \pm 0,48$ & $9-36$ & 143 & $-1,93$ & 0,05 \\
\hline Derechos & $3,53 \pm 0,4$ & $3,31 \pm 0,45$ & $9-36$ & 168,5 & $-1,4$ & 0,16 \\
\hline Bienestar emocional & $3,45 \pm 0,47$ & $3,18 \pm 0,49$ & $9-36$ & 152 & $-1,74$ & 0,08 \\
\hline Inclusión social & $3,46 \pm 0,42$ & $3,29 \pm 0,4$ & $9-36$ & 170 & $-1,36$ & 0,17 \\
\hline Desarrollo personal & $3,51 \pm 0,18$ & $3,15 \pm 0,41$ & $9-36$ & 113 & $-2,57$ & 0,01 \\
\hline Relaciones interpersonales & $3,22 \pm 0,57$ & $3,02 \pm 0,52$ & $9-36$ & 169 & $-1,39$ & 0,16 \\
\hline Bienestar material & $3,77 \pm 0,17$ & $3,65 \pm 0,22$ & $9-36$ & 157,5 & $-1,65$ & 0,09 \\
\hline Bienestar físico & $3,85 \pm 0,13$ & $3,59 \pm 0,33$ & $9-36$ & 104,5 & $-2,76$ & 0,006 \\
\hline
\end{tabular}

*Nota: $\mathrm{M}=$ Media; DT = Desviación típica; $\mathrm{SD}=$ Síndrome de Down. 


\section{LA “VENTAJA DEL SÍNDROME DE DOWN” EN LA CALIDAD DE VIDA DE JÓVENES CON DISCAPACIDAD INTELECTUAL ANA BERÁSTEGUI Y SARA CORRAL}

\subsection{CVI-A en jóvenes con y $\sin S D$}

Con relación a la calidad de vida individual autoinformada, los jóvenes con DI que no tienen SD presentan una mayor CVI que aquellos con SD, siendo estas diferencias significativas y el tamaño del efecto moderado $(U=-2,41 ; p=0,01 ; r=0,32)$ (ver Tabla 4).

Las dimensiones de la CV-A s en las que hubo diferencias fueron los derechos $(U=-2,56 ; p=0,01 ; r=0,34)$, la inclusión social $(U=2,29 ; p=0,02 ; r=0,30)$, las relaciones interpersonales $(U=2,21 ; p=0,02 ; r=0,29)$ y el bienestar material $(U=1,98$; $p=0,04 ; r=0,26)$, siendo las medias siempre superiores en el grupo de jóvenes con DI sin SD respecto al grupo con SD y los tamaños del efecto moderados o pequeños.

\begin{tabular}{|c|c|c|c|c|c|c|}
\hline \multicolumn{7}{|c|}{$\begin{array}{l}\text { TABLA 4. U de Mann Whitney, media y desviación típica de la calidad de vida } \\
\text { individual autoinformada }\end{array}$} \\
\hline & $\mathrm{SD}$ & No SD & \multirow{2}{*}{ Rango } & \multirow{2}{*}{$U$} & \multirow{2}{*}{$Z$} & \multirow{2}{*}{$p$} \\
\hline & $M \pm D T$ & $M \pm D T$ & & & & \\
\hline Calidad de vida & $3,03 \pm 0,4$ & $3,26 \pm 0,3$ & $72-288$ & 254,5 & $-2,41$ & 0,01 \\
\hline Autodeterminación & $3,02 \pm 0,46$ & $3,05 \pm 0,44$ & $9-36$ & 389 & $-0,5$ & 0,61 \\
\hline Derechos & $2,77 \pm 0,53$ & $3,15 \pm 0,48$ & $9-36$ & 244,5 & $-2,56$ & 0,01 \\
\hline Bienestar emocional & $3,16 \pm 0,49$ & $3,18 \pm 0,39$ & $9-36$ & 421 & $-0,04$ & 0,96 \\
\hline Inclusión social & $2,95 \pm 0,46$ & $3,27 \pm 0,42$ & $9-36$ & 263,5 & $-2,29$ & 0,02 \\
\hline Desarrollo personal & $3,34 \pm 0,38$ & $3,33 \pm 0,38$ & $9-36$ & 416,5 & $-0,1$ & 0,91 \\
\hline Relaciones interpersonales & $2,93 \pm 0,52$ & $3,26 \pm 0,46$ & $9-36$ & 248 & $-2,21$ & 0,02 \\
\hline Bienestar material & $3,33 \pm 0,43$ & $3,57 \pm 0,34$ & $9-36$ & 259 & $-1,98$ & 0,04 \\
\hline Bienestar físico & $3,39 \pm 0,39$ & $3,42 \pm 0,45$ & $9-36$ & 376,5 & $-0,204$ & 0,83 \\
\hline
\end{tabular}

*Nota: $M=$ Media; $S D=$ Desviación típica; $\mathrm{SD}=$ Síndrome de Down.

\subsection{Efecto de los ingresos y edad en la calidad de vida}

No se observa una relación entre la calidad de vida familiar y los ingresos familiares $[F(0,19,1)=-0,65>0,01]$ o la edad parental $[F(0,76,1)=-0,38>0,01]$. Tampoco se encuentra una relación entre la CV-A y los ingresos $[F(3,23,1)=-0,078>0,01]$ o ni con la edad parental $[F(0,05,1)=-0,81>0,01]$. Finalmente, no se encuentra una relación entre la $\mathrm{CV}-\mathrm{O}$ y los ingresos $[F(0,22,1)=-0,88>0,01]$ o la edad parental $[F$ $(0,95,1)=-0,33>0,01]$.

\section{Discusión}

Los hallazgos encontrados sugieren la existencia de una mayor satisfacción con la calidad de vida familiar en las familias de jóvenes con SD frente a las de aquellos con 
otra DI, especialmente en la subescala de apoyos y servicios, siendo estas diferencias moderadas. Estos resultados apoyan la explicación social de la "ventaja del SD”. Una visión social más positiva sobre el SD puede estar repercutiendo en un mayor apoyo social para estas familias, lo que se refleja en la mayor satisfacción de estas familias con los recursos y apoyos disponibles en nuestra muestra (Hodapp, 2007; Lee et al., 2018; Poehlmann et al., 2005). Sin embargo, en la investigación cualitativa reciente, el apoyo social forma parte muy relevante de la experiencia cotidiana de las familias de niños con SD, pero también lo hace su ausencia, con lo que no podemos desatender la posible presencia de estigmatización en este colectivo (Farkas, Cless, Cless, Goff, Bodine y Edelman, 2018).

Los resultados obtenidos en relación con la calidad de vida individual resultan discordantes ya que, cuando informan los familiares, las puntuaciones son mejores para el grupo de jóvenes con SD, mientras que, cuando son ellos mismos quienes informan, la relación se invierte y es mejor la CV del grupo de jóvenes con DI sin SD. Por otro lado, estas diferencias no se presentan de forma homogénea en todas las subescalas de la calidad de vida individual. Los progenitores de hijos con SD informan de mejores niveles de calidad de vida en el desarrollo personal, el bienestar físico y el bienestar emocional de sus hijos frente a los padres de sus compañeros sin SD. Hay que tener en cuenta el fuerte carácter subjetivo de estas escalas. Por su parte, los jóvenes con SD informan de peores niveles de derechos, integración social, relaciones interpersonales y bienestar material que lo que lo hacen sus compañeros sin SD, escalas con un carácter algo más objetivo que las primeras. Las diferencias son siempre moderadas o pequeñas.

En este caso, los progenitores han podido atribuir una mejor calidad de vida a sus hijos con SD que la que atribuyen los demás padres a sus hijos y que la que se atribuyen ellos mismos. Esta atribución positiva puede deberse al estereotipo positivo que acompaña al fenotipo conductual en SD (Rodgers, 2008), que les hace atribuirles un mayor nivel de bienestar, felicidad y desarrollo que el resto de los progenitores.

La presencia de atribuciones positivas, que puede impulsar un afrontamiento positivo en la familia, podría también estar escondiendo cierto sufrimiento y malestar percibido en el propio joven con SD. En contraste con el mayor apoyo social de estas familias, es precisamente en la inclusión social y las relaciones interpersonales, junto con los derechos y el bienestar material, donde los jóvenes con SD perciben una menor calidad de vida, frente a sus compañeros, así que el mayor apoyo que perciben sus familias no se traslada a la autopercepción de sus hijos.

En la mayoría de los estudios sobre esta cuestión, los informantes han sido los familiares de la persona con DI. La versión “informe de otras personas” puede dar una información útil, pero no debe sustituir la versión "autoinforme” cuando la persona puede informar sobre sí misma, ya que solo esta última puede ofrecernos una visión personal de la realidad. Por tanto, aunque sea conveniente contar con la percepción familiar, resulta fundamental utilizar medidas que evalúen directamente a las personas con discapacidad, especialmente en variables como la calidad de vida, que tienen un componente de bienestar subjetivo central. En cualquier caso, hay que tener también en cuenta la menor fiabilidad de la prueba INICO-FEAPS para la 


\section{LA “VENTAJA DEL SÍNDROME DE DOWN” EN LA CALIDAD DE VIDA DE JÓVENES CON DISCAPACIDAD INTELECTUAL ANA BERÁSTEGUI Y SARA CORRAL}

versión autoinformada (Verdugo et al., 2013), lo que puede estar empañando estos resultados. Por otro lado, sería interesante evaluar a los progenitores por separado, para ver si esta percepción se mantiene tanto para el padre como para la madre, e incluir la visión de los hermanos.

Finalmente, esta investigación no encuentra relación entre las dos variables identificadas en la literatura como factores de confusión principales -edad parental e ingresos- y las variables estudiadas. Respecto a la edad parental, en este estudio se contó indistintamente con la edad del padre o de la madre, dependiendo de quién contestara la prueba, mientras que la mayoría de las investigaciones atienden solo a la edad de la madre (por ejemplo, Urbano y Hodapp, 2007). Respecto a la variable ingresos, el sesgo socioeconómico de la muestra puede explicar la diferencia con otros estudios (por ejemplo, Stoneman, 2007).

Este estudio presenta algunas limitaciones con respecto a la muestra que deben ser tenidas en cuenta al interpretar los resultados. En primer lugar, todas las personas de la muestra forman parte del programa DEMOS de formación postobligatoria en la universidad (Berástegui, García y López-Riobóo, 2015). Aunque no se controlan las diferencias en inteligencia o habilidades adaptativas, el proceso de selección introduce un sesgo en cuanto a estas variables, con lo que los hallazgos tendrían que ser llevados a muestras más representativas de la DI y el SD. Por otro lado, no contamos con grupos suficientemente grandes como para comparar por etiologías. Además, el tamaño de los grupos no era muy grande y las submuestras estaban descompensadas, lo que limita el poder estadístico para detectar las diferencias.

Asimismo, se ha usado un diseño transversal para dar respuesta a una variable que no parece estable en el tiempo (Glidden et al., 2014; Hodapp, 2007). El aumento en la esperanza de vida de las personas con SD (Huete, 2016) nos alienta a estudiar esta cuestión más allá de la juventud, en la vida adulta y el proceso de envejecimiento, idealmente mediante un diseño longitudinal (Hodapp, 2007).

A pesar de estas limitaciones, el presente estudio representa un paso importante en la comprensión de la "ventaja del SD", ya que se han analizado desde esta perspectiva variables de CVI que no habían sido estudiadas incluidas en los estudios anteriores. Tras la presente investigación, se ve necesario un análisis más complejo de este fenómeno, resaltando la importancia de incluir en la medida de lo posible la autoevaluación de la calidad de vida, junto con el informe de otras personas. Por otro lado, el impacto positivo del conocimiento y el apoyo social hacia el SD en la familia nos coloca frente al reto de mejorar el conocimiento y la sensibilización de la sociedad con respecto a otras etiologías y discapacidades, y el desarrollo de recursos de apoyo social, lo que podría ayudar, en gran medida, a mejorar la calidad de vida de estas familias.

\section{Referencias bibliográficas}

Abbeduto, L., Seltzer, M., Shattuck, P., Krauss, M., Orsmond, G. y Murphy, M. (2004). Psychological well-being and coping in mothers of youths with autism, Down syndrome, or Fragile X syndrome. American Journal on Mental Retardation, 109(3), 237. http://doi. org/10.1352/0895-8017(2004)109<237:PWACIM>2.0.CO;2 


\section{LA “VENTAJA DEL SÍNDROME DE DOWN” EN LA CALIDAD DE VIDA DE JÓVENES CON DISCAPACIDAD INTELECTUAL \\ ANA BERÁSTEGUI Y SARA CORRAL}

Acharya, K. (2011). Prenatal testing for intellectual disability: misperceptions and reality with lessons from Down syndrome. Developmental Disabilities Research Reviews, 17(1), 27-31. http://doi.org/10.1002/ddrr.135

Berástegui, A., López-Riobóo, E. y García, N. (2015). Proyecto DEMOS. Una apuesta por la formación de las personas con discapacidad intelectual en el entorno universitario. En D. Cabezas y J. Flórez (Dirs.), Educación postsecundaria en entorno universitario para alumnos con discapacidad intelectual: Experiencias y resultados (pp. 31-44). Santander: Fundación Iberoamericana Down21.

Bertoli, M., Biasini, G., Calignano, M., Celani, G., De Grossi, G., Digilio, M. et al. (2011). Needs and challenges of daily life for people with Down syndrome residing in the city of Rome, Italy. Journal of Intellectual Disability Research, 55(8), 801-820. http://doi. org/10.1111/j.1365-2788.2011.01432.x

Blacher, J., Baker, B. L. y Kaladjian, A. (2013). Syndrome specificity and mother-child interactions: examining positive and negative parenting across contexts and time. Journal of Autism and Developmental Disorders, 43(4), 761-774. http://doi.org/10.1007/s10803-012-1605-x

Blacher, J. y McIntyre, L. L. (2006). Syndrome specificity and behavioural disorders in young adults with intellectual disability: cultural differences in family impact. Journal of Intellectual Disability Research, 50(3), 184-198. https://doi.org/0.1111/j.1365-2788.2005.00768.x

Brown, R., MacAdam-Crisp, J., Wang, M. y Iarocci, G. (2006). Family quality of life when there is a child with a developmental disability. Journal of Policy and Practice in Intellectual Disabilities, 3(4), 238-245. https://doi.org/10.1111/j.1741-1130.2006.00085.x

CoHen, J. (1988). Statistical power analysis for the behavioral sciences, 2nd Edition. Hillsdale: Lawrence Erlbaum.

Corrice, A. y Glidden, L. (2009). The Down syndrome advantage: fact or fiction? American Journal on Intellectual and Developmental Disabilities, 114(4), 254-268. http://doi.org/10.1352/1944-7558-114.4.254-268

Dabrowska, A. y Pisula, E. (2010). Parenting stress and coping styles in mothers and fathers of pre-school children with autism and Down syndrome. Journal of Intellectual Disability Research, 54(3), 266-280. https://doi.org/10.1111/j.1365-2788.2010.01258.x

Dodd, D. H., Zabriskie, R. B., Widmer, M. A. y Eggett, D. (2009). Contributions of family leisure to family functioning among families that include children with developmental disabilities. Journal of Leisure Research, 41(2), 261-286. https://doi.org/10.1080/00222216.2 009.11950169

Esbensen, A., Bishop, S., Seltzer, M., Greenberg, J. y Taylor, J. (2010). Comparisons between individuals with Autism Spectrum Disorders and individuals with Down syndrome in adulthood. American Journal on Intellectual and Developmental Disabilities, 115(4), 277-290. http://doi.org/10.1352/1944-7558-115.4.277

Esbensen, A. y Seltzer, M. (2011). Accounting for the "Down syndrome advantage". American Journal on Intellectual and Developmental Disabilities, 116(1), 3-15. http://doi. org/10.1352/1944-7558-116.1.3

Farkas, L., Cless, J. D., Clesss, A. W., Nelson Goff, B. S., Bodine, E. y Edelman, A. (2019). The ups and Downs of Down syndrome: a qualitative study of positive and negative parenting experiences. Journal of Family Issues, 40(4), 518-539. https://doi. org/10.1177/0192513X18812192

Fidler, D. J., Hodapp, R. M. y Dykens, E. M. (2000). Stress in families of young children with Down syndrome, Williams syndrome, and Smith-Magenis syndrome. Early Education and Development, 11(4), 395-406. https://doi.org/10.1207/s15566935eed1104_2 


\section{LA “VENTAJA DEL SÍNDROME DE DOWN” EN LA CALIDAD DE VIDA DE JÓVENES CON DISCAPACIDAD INTELECTUAL \\ ANA BERÁSTEGUI Y SARA CORRAL}

Fisman, S., Wolf, L., Ellison, D. y Freeman, T. A. (2000). Longitudinal study of siblings of children with chronic disabilities. The Canadian Journal of Psychiatry, 45(4), 369-375. https://doi.org/10.1177\%2F070674370004500406

Foley, K. R., Taffe, J., Bourke, J., Einfeld, S. L., Tonge, B. J., Trollor, J. y Leonard, H. (2016). Young people with intellectual disability transitioning to adulthood: do behaviour trajectories differ in those with and without Down syndrome? PloS one, 11(7), 1-14. https://doi.org/10.1371/journal.pone.0157667

Glidden, L., Grein, K. y Ludwig, J. (2014). The Down syndrome advantage: it depends on what and when you measure. American Journal on Intellectual and Developmental Disabilities, 119(5), 389-404. https://doi.org/10.1352/1944-7558-119.5.389

Griffith, G. M., Hastings, R. P., Nash, S. y Hill, C. (2010). Using matched groups to explore child behavior problems and maternal well-being in children with Down syndrome and autism. Journal of Autism and Developmental Disorders, 40(5), 610-619. https://doi. org/10.1007/s10803-009-0906-1

Hartley, S. L., Seltzer, M. M., Head, L. y Abbeduto, L. (2012). Psychological well-being in fathers of adolescents and young adults with Down syndrome, Fragile X syndrome, and autism. Family Relations, 61(2), 327-342. https://doi.org/10.1111/j.1741-3729.2011.00693.x

Hodapp, R. (2007). Families of persons with Down syndrome: new perspectives, findings, and research and service needs. Mental Retardation and Developmental Disabilities Research Reviews, 13(3), 279-287. http://dx.doi.org/10.1002/mrdd.20160

Hodapp, R. M., Burke, M. M. y Urbano, R. C. (2012). What's age got to do with it? Implications of maternal age on families of offspring with Down syndrome. International Review of Research in Developmental Disabilities, 42, 109-145. https://doi.org/10.1016/B978-012-394284-5.00005-X

Hodapp, R. M., Ricci, L. A., Ly, T. M. y Fidler, D. J. (2003). The effects of the child with Down syndrome on maternal stress. British Journal of Developmental Psychology, 21(1), 137-151. https://doi.org/10.1348/026151003321164672

Huete, A. (2016). Demografía e inclusión social de las personas con síndrome de Down. Revista Sindrome de Down, 33, 38-50.

Kasari, C. y Freeman, S. F. (2001). Task-related social behavior in children with Down syndrome.JournalInformation, 106(3),253-264.https://doi.org/10.1352/0895-8017(2001)106<0253:tr sbic $>2.0 . c 0 ; 2$

Lee, C., Burke, M., Arnold, C. y Owen, A. (2018). Comparing differences in support needs as percieved by parents of adult offspring with Down syndome, Autism Spectrum Disorder an cerebral palsy. Journal of Applied Research in Intellectual Disabilities, 32(1), 194205. https://doi.org/10.1111/jar.12521

Mitchell, D. B., Hauser-Cram, P. y Crossman, M.K. (2015). Relationship dimensions of the "Down Sydrome Advantage". Journal of Intellectual Disability Research, 59(6), 506518. https://doi.org/10.1111/jir.12153

Park, J., Hoffman, L., Marquis, J., Turnbull, A. P., Poston, D., Mannan, H., Wang, M. y Nelson, L. L. (2003). Toward assessing family outcomes of service delivery: validation of a Family Quality of Life Survey. Journal of Intellectual Disability Research, 47(4/5), 367384. https://doi.org/10.1046/j.1365-2788.2003.00497.x

Phillips, B. A., Conners, F. y Curtner-Smith, M. E. (2017). Parenting children with Down syndrome: an analysis of parenting styles, parenting dimensions, and parental stress. Research in Developmental Disabilities, 68, 9-19. http://doi.org/10.1016/j.ridd.2017.06.010

Pisula, E. A. (2007). Comparative study of stress profiles in mothers of children with autism and those of children with Down's syndrome. Journal of Applied Research in Intellectual Disabilities, 20(3), 274-278. http://doi.org/10.1111/j.1468-3148.2006.00342.x

Ediciones Universidad de Salamanca / CC BY-NC-ND

Siglo Cero, vol. 51 (4), 2020, octubre-diciembre, pp. 39-51 


\section{LA “VENTAJA DEL SÍNDROME DE DOWN” EN LA CALIDAD DE VIDA DE JÓVENES CON DISCAPACIDAD INTELECTUAL ANA BERÁSTEGUI Y SARA CORRAL}

Poehlmann, J., Clements, M., Abbeduto, L. y Farsad, V. (2005). Family experiences associated with a child's diagnosis of fragile X or down syndrome: evidence for disruption and resilience. Mental Retardation, 43(4), 255-267. http://dx.doi.org/10.1352/00476765(2005)43[255:FEAWAC]2.0.CO;2

Ricci, L. A. y Hodapp, R. M. (2003). Fathers of children with Down's syndrome versus other types of intellectual disability: perceptions, stress and involvement. Journal of Intellectual Disability Research, 47(4-5), 273-284. https://doi.org/10.1046/j.1365-2788.2003.00489.x

Rodgers, C. (2008). Maternal support for the down's syndrome stereotype: the effect of direct experience of the condition. Journal of Intellectual Disability Research, 31(3), 271-278. https://doi.org/10.1111/j.1365-2788.1987.tb01370.x

Rosner, B. A., Hodapp, R. M., Fidler, D. J., Sagun, J. N. y Dykens, E. M. (2004). Social competence in persons with Prader-Willi, Williams and Down's syndromes. Journal of Applied Research in Intellectual Disabilities, 17(3), 209-217. https://doi.org/10.1111/ j.1468-3148.2004.00200.x

Schalock, R. L., Keith, K. D., Verdugo, M. Á. y Gómez, L. E. (2010). Quality of life model development in the field of intellectual disability. En R. Kober, Quality of life for people with intellectual disability (pp. 17-32). Nueva York: Springer.

Schalock, R. L. y Verdugo, M. Á. (2012). A leadership guide to redefining intellectual and developmental disabilities organizations: eight successful change strategies. Baltimore, MD: Brookes Publishing Company.

Shivers, C. M., McGregor, C. y Hough, A. (2019). Self-reported stress among adolescent siblings of individuals with autism spectrum disorder and Down syndrome. Autism, 23(1), 112-122. https://doi.org/10.1177\%2F1362361317722432

Stoneman, Z. (2007). Examining the Down syndrome advantage: mothers and fathers of young children with disabilities. Journal of Intellectual Disability Research, 51(12), 10061017. http://doi.org/10.1111/j.1365-2788.2007.01012.x

Tekinarslan, I. A. (2013). Comparison study of depression and quality of life in Turkish mothers of children with Down syndrome, cerebral palsy, and Autism Spectrum Disorder. Psychological Reports, 112(1), 266-287. http://doi.org/10.2466/21.02.15.PR0.112.1.266-287

Tomczak, M. y Tomcak, E. (2014). The need to report effect size estimates revisited. An overview of some recommended measures of effect size. Trends Sport Sciences, 1(21), 19-25.

Urbano, R. y Hodapp, R. (2007). Divorce in families of children with Down syndrome: a population-based study. American Journal on Mental Retardation, 112(4), 261. http://doi. org/10.1352/0895-8017(2007)112[261:DIFOCW]2.0.CO;2

Van Gameren-Oosterom, H., Fekkes, M., Buitendijk, S., Mohangoo, A., Bruil, J. y Van Wouwe, J. (2011). Development, problem behavior, and quality of life in a population based sample of eight-year-old children with down syndrome. PLoS ONE, 6(7), e21879. http://doi.org/10.1371/journal.pone.0021879

Verdugo, M. Á., Gómez, L. E., Arias, B., Santamaría, M., Clavero, D. y Tamarit, J. (2013). Escala INICO-FEAPS: Evaluación Integral de la Calidad de Vida de Personas con Discapacidad Intelectual o del Desarrollo. Salamanca: Publicaciones INICO. https://sid. usal.es.

Verdugo, M. Á., Rodríguez, A. y SAinz, F. (2009). Escala de Calidad de Vida Familiar. Salamanca: Publicaciones INICO. https://sid.usal.es.

Wiley, R. y Renk, K. (2007). Psychological correlates of quality of life in children with cerebral palsy. Journal of Developmental and Physical Disabilities, 19(5), 427-447. http://doi. org /10.1007/s10882-007-9041-0 


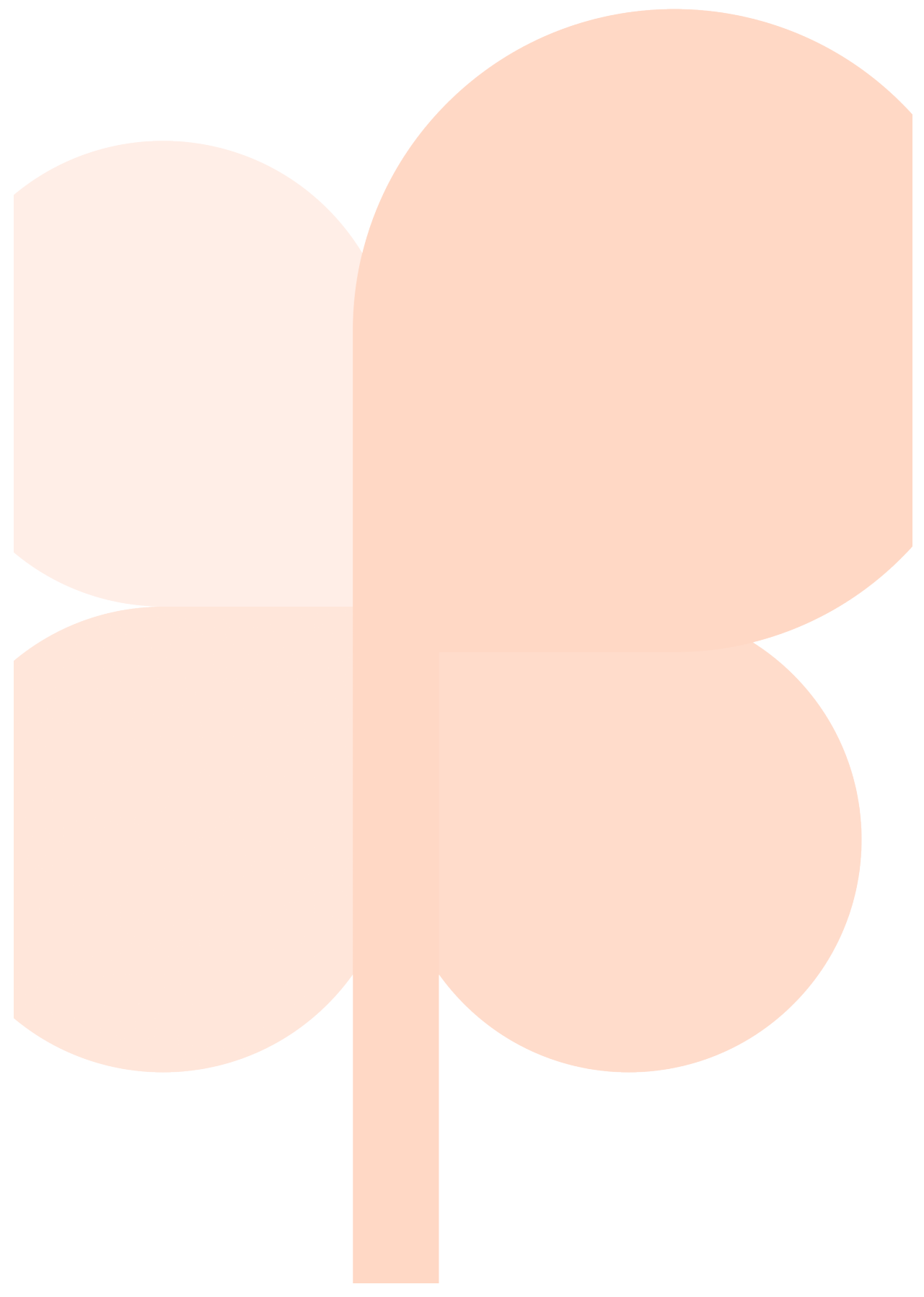

